\title{
Parallel Fabry-Pérot interferometer in suspended twin-core fiber
}

\author{
Ricardo M. André ${ }^{\mathrm{a}, \mathrm{b}^{*}}$, Jens Kobelke ${ }^{\mathrm{c}}$, Kay Schuster ${ }^{\mathrm{c}}$, Hartmut Bartelt ${ }^{\mathrm{c}}$, Manuel B. Marques ${ }^{\mathrm{a}, \mathrm{b}}$, \\ Orlando Frazão ${ }^{\text {a }}$ \\ ${ }^{a}$ INESC Porto, Rua do Campo Alegre 687, 4169-007 Porto, Portugal; \\ ${ }^{\text {b}}$ Departamento de Física da Faculdade de Ciências da Universidade do Porto, Rua do Campo Alegre \\ 687, 4169-007 Porto, Portugal \\ ${ }^{\mathrm{c}} \mathrm{IPHT}$, Institute of Photonic Technology, Jena, Albert-Einstein-Str. 9, 07745 Jena, Germany
}

\begin{abstract}
Small sections of suspended twin-core fiber are used in reflection configurations to create two parallel Fabry-Pérot cavities. Situations where both cores are excited and where only one core is excited are analyzed and compared. When both cores are excited, two parallel and equivalent cavities are formed and an interference pattern with higher visibility is obtained. The structure is also characterized with respect to temperature and a sensitivity of $12.4 \mathrm{pm} / \mathrm{K}$ is achieved.
\end{abstract}

Keywords: optical fiber sensors, suspended twin-core fiber, Fabry-Pérot interferometer, temperature sensing

\section{INTRODUCTION}

Twin-core fibers, also known as dual-core or two-core fibers, were first proposed as strain sensors in 1981 [1]. Their intrinsic coupling properties lead to femtosecond all-optical switching nonlinear couplers [2]. In optical sensing, temperature sensors were demonstrated [3]. With the advent of Photonic Crystal Fibers (PCF), several twin core fibers were developed such as twin-core fibers with alveolar air cladding [4] and twin-core photonic bandgap fibers [5]. Hybrid twin core fibers where light is guided by total internal reflection in one core and by bandgap guidance in the other were also proposed for wavelength-selective coupling [6]. More recently another type of microstructured twin-core fiber was developed, the suspended twin-core fiber was developed. A suspended core fiber is one where the core appears to be suspended in large air holes by thin glass bridges [7]. In the suspended twin-core fiber (STCF) case, the holes are distributed in order to create two triangular suspended cores. This kind of fiber has been applied as an all-fiber MachZehnder for temperature and strain-independent torsion sensing [8] and for temperature, strain and curvature discrimination [9].

In this work, a point-sensor based on a Fabry-Pérot cavity is presented for temperature sensing. A small section of suspended twin-core fiber is spliced to standard single mode fiber. Two cases are compared, one where both cores are illuminated and one where only one is illuminated. The interference pattern relative to the optical path difference between light reflected at the splice and reflected at the fiber end is monitored. The structure is then characterized with respect to temperature variations by monitoring the spectral shifts.

\section{FABRICATION AND CHARACTERIZATION}

The sensing heads were produced by splicing a section of STCF to standard SMF. The suspended twin-core fiber used has four holes and was fabricated at IPHT (Institute of Photonic Technology, Jena). The cores have an in circle diameter of $1.5 \mu \mathrm{m}$ and a spacing of $7.6 \mu \mathrm{m}$. Since this distance is large and the connecting bridge is very thin $(\sim 0.6 \mu \mathrm{m})$, little or no coupling is expected. In order to excite both cores equally or just one core, a fiber inspection microscope was used while aligning the SMF and STCF in the arc fusion splice machine. The arc discharge with low current was applied on the SMF side and not centered between SMF and STCF [10]. This way, the risk of hole collapse or core recoil is minimized.

\footnotetext{
*randre@inescporto.pt
} 
The setup used to characterize this sensing head is depicted in Figure 1. The sensing head is illuminated with an erbiumdoped optical broadband source with a central wavelength of $1550 \mathrm{~nm}$ and a spectral width of $100 \mathrm{~nm}$. Reflected light is collected, with the aid of an optical circulator, by an optical spectrum analyzer with a maximum resolution of $0.01 \mathrm{~nm}$.

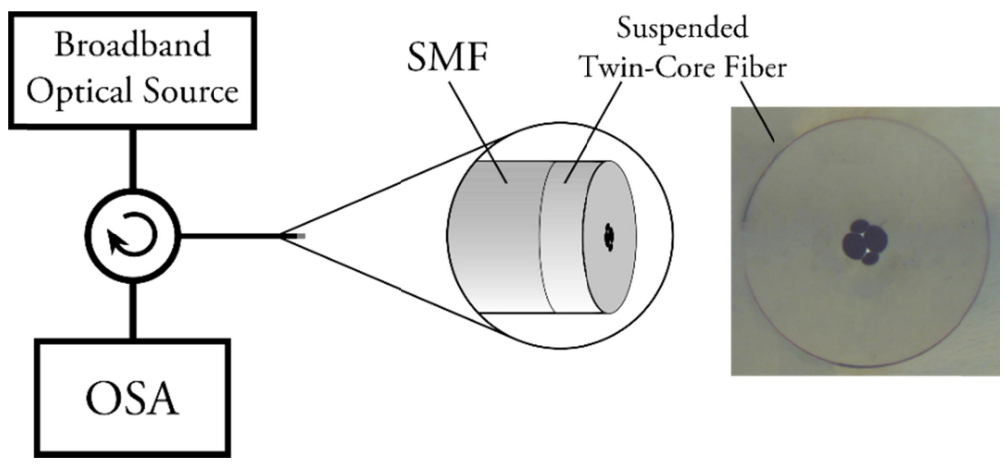

Figure 1. Experimental setup of sensing head characterization. Inset: fiber tip with section of suspended twin-core fiber.

\section{RESULTS AND DISCUSSION}

Several sensing heads with different STCF lengths $[30,20,13,5,1] \mathrm{mm}$ were analyzed. The typical spectral response is a fringe pattern as depicted in Figure 2. Since there is little to no coupling between cores, much less at these very small STCF lengths the interference comes from the different optical path lengths of light reflected at the splice and at the cleaved end. This can effectively be described as a Michelson interferometer or a low finesse Fabry-Pérot cavity. Light travels independently in both cores and is again recombined at the splice. The Fabry-Pérot cavity can be modeled considering the interference between two beams using the following equation:

$$
I=I_{1}+I_{2}+2 \sqrt{I_{1} I_{2}} \cos \left(\frac{2 \pi}{\lambda_{0}} \cdot O P D+\phi_{0}\right)
$$

where I is the intensity of the interference, $\phi_{0}$ is the initial phase and $\lambda_{0}$ is the operating wavelength in vacuum. The OPD of a round-trip is given by: OPD $=2 n_{\text {core }} L$, where in this case, $L$ is the length of the STCF section and is the same in both cases and $n_{\text {core }}$ is the cores' refractive index. As seen in Figure 2, the reflection spectrum shows an interference pattern with a fringe visibility of approximately $4 \mathrm{~dB}$, enough for most sensing applications. The spectrum from the 5 $\mathrm{mm}$ sensing head with both cores is represented.
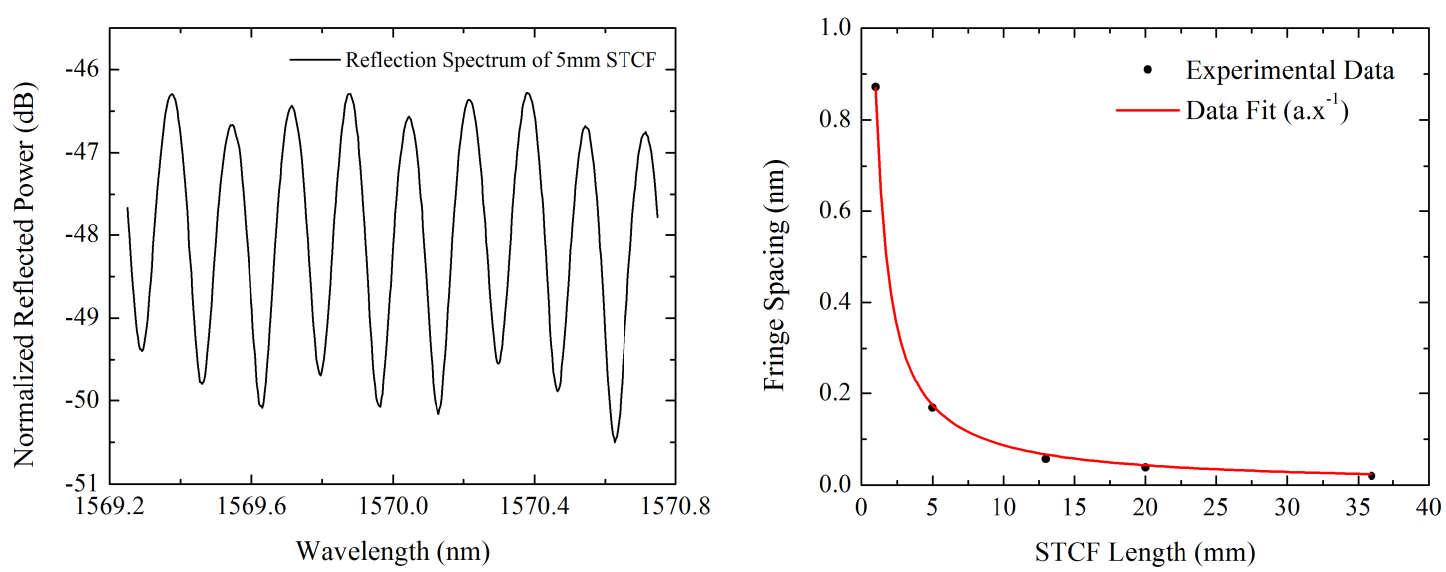

Figure 2. Reflection spectrum of the $5 \mathrm{~mm}$ STCF sensing head (left) and fringe spacing as a function of STCF length (right). 
Comparing the fringe spacing of the spectra for the different sensing head lengths, a decrease of the spacing is observed with increasing STCF length this is expected from the following equation that can be derived from the optical path length difference:

$$
\Delta \lambda=\frac{\lambda^{2}}{2 n_{\text {core }}} \cdot \frac{1}{L}
$$

Sensing heads with $13 \mathrm{~mm}$-STCF sections read in reflection but where either both cores are illuminated or just one core is excited are compared in Figure 3. Readily observable is the difference in reflected optical power and visibility. While in the case where only one core is excited there is much more power, in the case where both cores are illuminated, there is much higher visibility. Higher power in the case where only one core is excited is expected since the mode field of the SMF is centered with one of the STCF cores, while in the other case, to excite both cores equally, the SMF mode field is centered between the cores where only air and a thin bridge exists. When both cores are excited, an interference pattern with a higher visibility is created due to sum of the in-phase signals that originate from both cores.

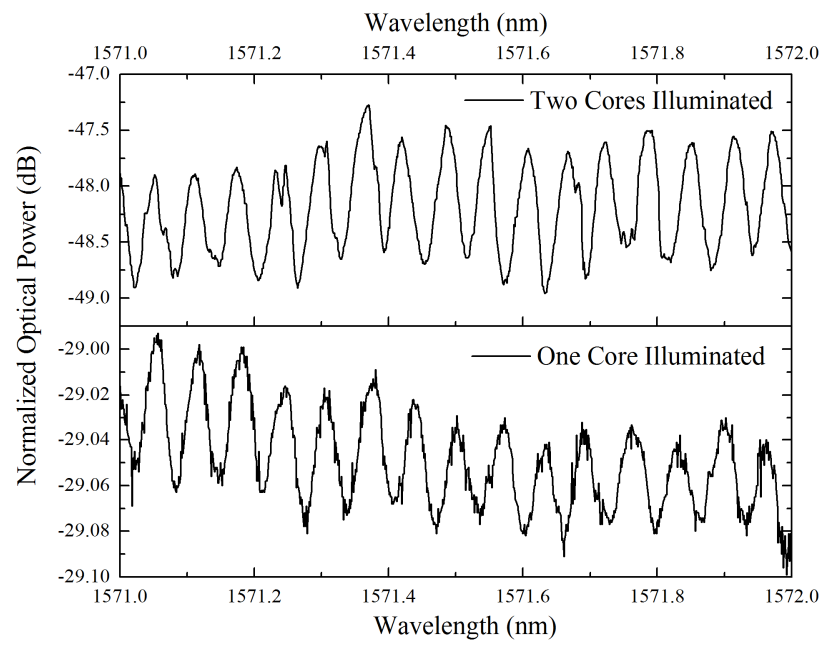

Figure 3. Reflection spectra of $13 \mathrm{~mm}$ STCF sections where both cores are illuminated (top) and only one core is illuminated (bottom).

To demonstrate the capability of this structure as a high temperature sensor, the $5 \mathrm{~mm}$ sensing head was placed in an electric furnace and characterized with respect to temperature variations in the range $100-500{ }^{\circ} \mathrm{C}$. A linear regression was used to fit the response $\left(\mathrm{R}^{2}=0.9995\right)$ and a sensitivity of $12.4 \mathrm{pm} / \mathrm{K}$ was calculated (see Figure 4$)$. The temperature sensitivity is in line with that obtained by others for similar suspended core fiber and suspended twin-core fiber structures (see Table 1).

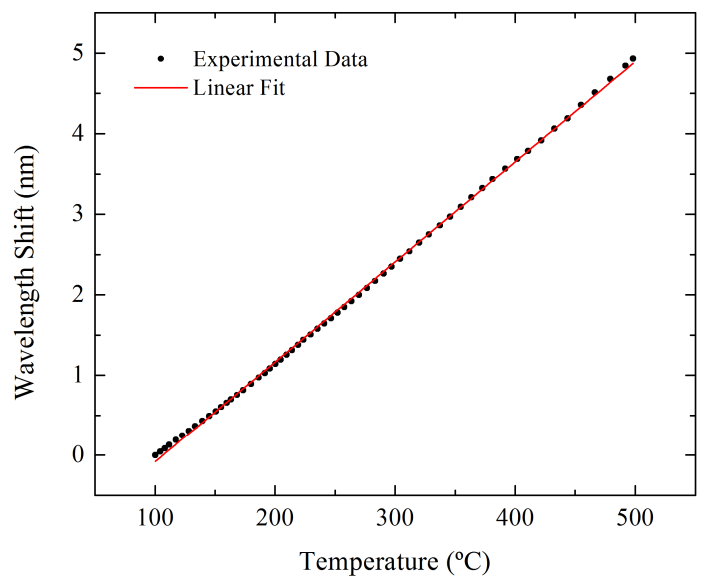

Figure 4. Wavelength shift with temperature variation. Temperature sensitivity of $12.4 \mathrm{pm} / \mathrm{K}$ and a coefficient of determination $\mathrm{R}^{2}=0.9995$. 
Table 1. Temperature sensitivity comparison in literature.

Fiber and Mechanism

Suspended twin-core fiber-parallel FP interferometer

Suspended twin-core fiber-Mach-Zehnder interferometer

Suspended twin-core fiber-Fiber Loop Mirror

Suspended core fiber - Fabry-Pérot cavity
Temperature Sensitivity Reference

This work

$7.65 \mathrm{pm} / \mathrm{K}$ (3-hole SCF)

$8.89 \mathrm{pm} / \mathrm{K}$ (4-hole SCF)

\section{CONCLUSIONS}

To summarize, suspended twin-core fiber was used to fabricate point sensors where a small section is spliced to SMF. An analysis of the interference pattern as a function of the STCF length was performed. As expected, a larger fringe spacing was obtained for smaller sensing heads. A temperature sensitivity $12.4 \mathrm{pm} / \mathrm{K}$ obtained for the $5 \mathrm{~mm}$ STCFsection sensing head is in accordance with results obtained in literature. Situations where both cores or only one core were illuminated were compared. When both cores are illuminated a higher visibility is achieved and this could be interesting for refractive index measurements using these structures as dip sensors.

\section{REFERENCES}

[1] G. Meltz and E. Snitzer, "Fiber optic strain sensor," Google Patents (1981).

[2] S. R. Friberg, A. M. Weiner, Y. Silberberg, B. G. Sfez, and P. S. Smith, "Femtosecond switching in a dual-core-fiber nonlinear coupler," Optics Letters 13(10), 904 (1988).

[3] G. Meltz, J. R. Dunphy, W. W. Morey, and E. Snitzer, "Cross-talk fiber-optic temperature sensor," Applied Optics 22(3), 464 (1983).

[4] L. Zhang and C. Yang, "Polarization-Dependent Coupling in Twin-Core Photonic Crystal Fibers," Journal of Lightwave Technology 22(5), 1367-1373 (2004).

[5] Z. Wang, T. Taru, T. A. Birks, J. C. Knight, Y. Liu, and J. Du, "Coupling in dual-core photonic bandgap fibers: theory and experiment," Optics Express 15(8), 4795 (2007).

[6] X. Sun, "Wavelength-selective coupling of dual-core photonic crystal fiber with a hybrid light-guiding mechanism," Optics Letters 32(17), 2484 (2007).

[7] T. M. Monro, S. Warren-Smith, E. P. Schartner, A. François, S. Heng, H. Ebendorff-Heidepriem, and S. Afshar, "Sensing with suspended-core optical fibers," Optical Fiber Technology 16(6), 343-356 (2010).

[8] O. Frazão, S. F. O. Silva, J. Viegas, J. M. Baptista, J. L. Santos, J. Kobelke, and K. Schuster, "All Fiber MachZehnder Interferometer Based on Suspended Twin-Core Fiber," IEEE Photonics Technology Letters 22(17), 1300-1302 (2010).

[9] S. F. O. Silva, "Simultaneous measurement of three parameters using an all-fiber Mach-Zehnder interferometer based on suspended twin-core fibers," Optical Engineering 50(3), 030501 (2011).

[10] O. Frazão, J. P. Carvalho, and H. M. Salgado, "Low-loss splice in a microstructured fibre using a conventional fusion splicer," Microwave and Optical Technology Letters 46(2), 172-174 (2005).

[11] O. Frazão, R. M. Silva, J. Kobelke, and K. Schuster, "Temperature- and strain-independent torsion sensor using a fiber loop mirror based on suspended twin-core fiber," Optics Letters 35(16), 2777-2779 (2010).

[12] O. Frazão, S. H. Aref, J. M. Baptista, J. L. Santos, H. Latifi, F. Farahi, J. Kobelke, and K. Schuster, "Fabry-Pérot Cavity Based on a Suspended-Core Fiber for Strain and Temperature Measurement," IEEE Photonics Technology Letters 21(17), 1229-1231 (2009). 